\title{
BART: The Czech Autonomous Observatory
}

\author{
Martin Nekola, ${ }^{1}$ René Hudec, ${ }^{1}$ Martin Jelínek, ${ }^{2}$ Petr Kubánek, ${ }^{2,3}$ \\ Jan Štrobl, ${ }^{1}$ and Cyril Polášek ${ }^{1}$ \\ ${ }^{1}$ Astronomical Institute, The Academy of Sciences of the Czech Republic, Fričova 298, CZ-251 65 Ondřejov, Czech Republic \\ ${ }^{2}$ Instituto de Astrofísica de Andalucía (CSIC), Camino Bajo de Huétor, 50, 18.008 Granada, Spain \\ ${ }^{3}$ Laboratorio de Procesado de Imágenes, Parque Científico del Campus de Paterna, Institutos de Investigación, \\ Universidad de Valencia, edificio 3, planta 3, Polígono Industrial La Coma, s/n, 46.980 Paterna, Spain \\ Correspondence should be addressed to Martin Nekola, ford@asu.cas.cz
}

Received 30 June 2009; Revised 30 November 2009; Accepted 16 January 2010

Academic Editor: Alberto J. Castro-Tirado

Copyright () 2010 Martin Nekola et al. This is an open access article distributed under the Creative Commons Attribution License, which permits unrestricted use, distribution, and reproduction in any medium, provided the original work is properly cited.

The High Energy Astrophysics group of the stellar department of the Astronomical Institute in Ondřejov operates two small aperture robotic telescopes called BART and D50. Both telescopes are capable of making automatic followup observation of gamma-ray burst optical counterparts. This paper deals with the smaller telescope BART.

\section{Introduction}

An increasing use of microprocessors in different professions has also touched an astronomical instrumentation like a telescope guiding, or even controlling of a whole observatory including an observation scheduling. Nowadays there is roughly one hundred robotic telescopes throughout the world. A degree of robotization level differs. Only few of them reach the level of complete independence. Generally the term robotic telescope stand for any telescope using advanced information technologies - a computer is at least able to guide a telescope to given position and take images or is capable of more complicated tasks. The term autonomous observatory means a robotic telescope and dome are computer controlled in such manner, that all indispensable actions of observation are done automatically, including processing of weather conditions, dome driving, choosing objects to observe, exposing by cameras or other optical sensors, taking calibration images, and so forth. No human interaction nor activity is necessary for observation.

\section{BART}

The Burst Alert Robotic Telescope (BART) is an independent robotic observatory, employing an $0.25 \mathrm{~m}$ optical telescope, dedicated to monitoring of objects interesting for high energy astrophysics like X-ray stars, supernovae, active galactic nuclei (AGN), blazars, cataclysmic variable stars (CV), quasars and to Gamma-Ray Burst (GRB) follow-up.

BART has been since the beginning designed as an autonomous robotic observatory. Thanks to the effort of our team lasting few years we achieved the required level of automation. It is operated by High Energy Astrophysics group of the Stellar department of the Astronomical Institute in Ondřejov. It is the first autonomous observatory in the Institute and the first observatory controlled by Remote Telescope System (RTS) software [1-3].

The space telescopes and orbital observatories working in sundry energy ranges represent an important tool of modern astrophysics with many valuable inputs for the different fields of astronomy and astrophysics. There are scientific applications where the support by ground-based robotic telescopes can substantially improve the scientific value of satellite data. Examples of such objects are INTEGRAL gamma-ray sources, CV or blazars. Also simultaneous optical data for various satellite campaigns are needful. The groundbased telescopes with immediate response to satellite observations have been designed mostly for immediate optical GRB observations. The system can be applied also for other types of astrophysical objects requiring immediate groundbased observations, like flares of $\mathrm{TeV}$ sources or blazars. Our telescope BART can perform observing of objects without human interaction including immediate reaction to GCN (GRB Coordinates Network [4]) alert or long term 
monitoring of selected objects, for example GK Per, TT Ari, OJ 287.

Another application of robotic telescopes is for example. monitoring of various celestial objects for their activity states, like CV, AGN and blazars. It can then provide inputs for following satellite observations during active states of these sources ( $\mathrm{ToO}$ - targets of opportunity). Another possibility is a simultaneous observation providing complementary optical data as well as good sampling over long time interval, for example. INTEGRAL gamma-ray sources with optical counterparts where mostly the optical light curve is not available for comparison with gamma-ray light curves. The software RTS2 allows planning of observation of such objects with user defined priority and procedure of observation.

BART is made of devices easily available on market (mount, optical telescope assembly, CCD camera, focuser, filter wheel). It is relatively easy to build it and reproduce it.

BART was also serving as a platform for developing and testing of software package RTS. An advantage of BART is the availability of location from the capital Prague, where is located the Charles University. Students can come to observatory and learn how to observe with BART and how to process acquired data.

\section{History of BART}

Using of inexpensive instrumentation supplemented by dedicated software was first time considered in 1992. Further idea was to equip the telescope by attached wide field (WF) camera covering the error box of the BATSE satellite. There was also an additional idea of using the attached WF camera as a testing device. It had similar parameters to the optical monitoring camera (OMC) onboard the INTEGRAL17 arc seconds/pixel and limiting magnitude about 15 .

At the beginning we had $254 \mathrm{~mm}\left(10^{\prime \prime}\right)$ telescope Meade LX200 with fork mount, CCD cameras SBIG, Meopta lens f $109 \mathrm{~mm} / \mathrm{d} 82.5 \mathrm{~mm}$, PC, observing room, so we did not search ideal unique place with good weather, dark night sky and electric power, optical telescope assembly with the best quality optics and stable construction, stable and fast mount for used telescope capable of accurate positioning and tracking and guide cables from devices like cameras and so forth. We used what we had.

We needed to develop or to get many things like a controlling software, a computer controlled roof, weather sensors, Internet connectivity, an uninterruptable power source, photometric filters and a computer controlled carousel and a focuser and so forth. We faced to many problems, for example avoiding of collision of telescope with roof or other things in vicinity, broken cameras, guiding cables from cameras and so forth, bad optics fixing, dew at optics, stray light, weather data acquisition and processing, useful calibration images, logical archive system, data processing and reduction, and so on.

The first controlling software for telescope BART was developed by Jan Soldán. Software for controlling of autonomous observatory RTS was later developed by students of Faculty of Mathematics and Physics of Charles University in Prague, mainly by Petr Kubánek. Later it was rewritten.
The current version of RTS is number 2 (RTS2). During development of BART various approaches and methods were tested.

Observation began in 2001. BART was a robotic telescope capable of fast follow-up observation of GCN alerts. It was not yet an autonomous observatory. Man had to manually open and close the roof, switch on and off the mount, manually to do focusing, watch a weather conditions and check the controlling software. It is necessary never again. During BART's lifetime we changed CCD cameras, the mount, WF lenses, a focusing, photometric filters and software. BART later generated daughter experiments such as BOOTES and FRAM.

We plan to preprocess the data archive for a better accessibility of the data and provide a preview of a light curve of an examined object.

\section{A Contemporary BART Configuration}

BART consists of the following devices:

(i) optical telescope assembly: 10" Schmidt-Cassegrain Meade LX 200-an amateur astronomers classic,

(ii) FLI digital focuser,

(iii) color filter wheel FLI CFW-1,

(iv) CCD camera FLI MaxCam CM2-1 with backilluminated chip,

(v) equatorial mount of German type Losmandy Titan + Gemini GoTo guiding system,

(vi) wide field camera attached to the Meade: teleobjective Rubinar, CCD camera FLI IMG6303,

(vii) room with sliding roof at solar building; it can be opened remotely; room was inherited from another experiment; It was necessary to do some improvements,

(viii) precipitation detector Meteoservis RDM1,

(ix) cloud meter based on measurement of thermal radiation,

(x) Polaris watcher-CCD camera with small chip and Meopta lens f $109 \mathrm{~mm} / \mathrm{d} 82.5 \mathrm{~mm}$.

Main parameters of the telescope and the attached wide field camera you can find in the Table 1. BART is shown in the Figure 1.

\section{Software}

BART uses the following software:

(i) GNU/Linux: an operating system,

(ii) RTS-2: a GNU GPL licensed software package for controlling of autonomous observatory,

(iii) PostgreSQL: an open source SQL database.

RTS2 is an integrated open source package for remote telescope control running under the Linux operating system. It can control various cameras, photometers, mounts and 
TABLE 1: BART parameters.

\begin{tabular}{lll}
\hline & Telescope & WF camera \\
\hline optical setup & Schmidt-Cassegrain & Maksutov-Cassegrain \\
focal length & $1600 \mathrm{~mm}$ & $500 \mathrm{~mm}$ \\
aperture & $254 \mathrm{~mm}$ & $89 \mathrm{~mm}$ \\
field of view & $28 \times 28$ arc minutes & $3.1 \times 2.1$ arc degree \\
CCD sensor resolution & $1024 \times 1024$ of $13 \mu \mathrm{m}$ pixels & $3088 \times 2056$ of $9 \mu \mathrm{m}$ pixels \\
photometric filters & Johnson B, V, R, i, z & - \\
limiting magnitude & 17.5 at $3 \sigma$ for $60 \mathrm{~s}$ in R & 16.2 at $3 \sigma$ for $60 \mathrm{~s}$ \\
slew speed & 6 arc degrees $/ \mathrm{s}$ & - \\
\hline
\end{tabular}

dome controllers. RTS2 can perform a whole observation from choosing targets from a database, opening and closing roof, changing position of a telescope, setting photometric filters, taking exposures and calibration images by cameras, processing images and storing their coordinates and other data to the SQL database. RTS2 uses the FITS data format for storing images all time. Now it writes also coordinates in the FITS WCS (World Coordinate System) to the image header. FITS and WCS are well known standards.

Every target object in our database has its own identification number and observing script. The script describes the length and number of expositions and used photometric filters for one observation of an object. A number of observations can belong to one object.

RTS2 can choose targets during a night by calculating a merit function of all observable targets during every last exposition of actual script or can perform queue scheduling or direct entering of targets. There is also a module for receiving and processing of GCN alerts. It interrupts execution of actual script and guides the telescope to the coordinates received by the GCN alert message and starts the observation according to a script for GRBs.

RTS2 is capable of remote supervision by a secure shell (ssh) remote login and by a program rts2-mon, which is a part of the package allowing to see a state of all devices and daemons and to change selected state parameters of the running system. RTS2 also includes several utilities for adding new targets and their observing scripts to the SQL database, generating a list of targets stored in the database or showing information about selected target or image and for selecting images from archive according to entered criteria. In the past there was a website for accessing the database of targets and preview images using any web browser. There is an informative website about BART http://lascaux.asu.cas.cz/en.

\section{Science with BART}

GRBs are most energetic known explosions in the universe. BART has observed many GRB positions as a prompt reaction to GCN alerts. It had seen optical afterglows of GRB-GRB060218, GRB060512. Measurements of negative observations gave magnitudes, that were published at GCN circulars [5]. A part of them you can see in the Table 2. In the Table 3 you can see BART reactions to GCN alerts

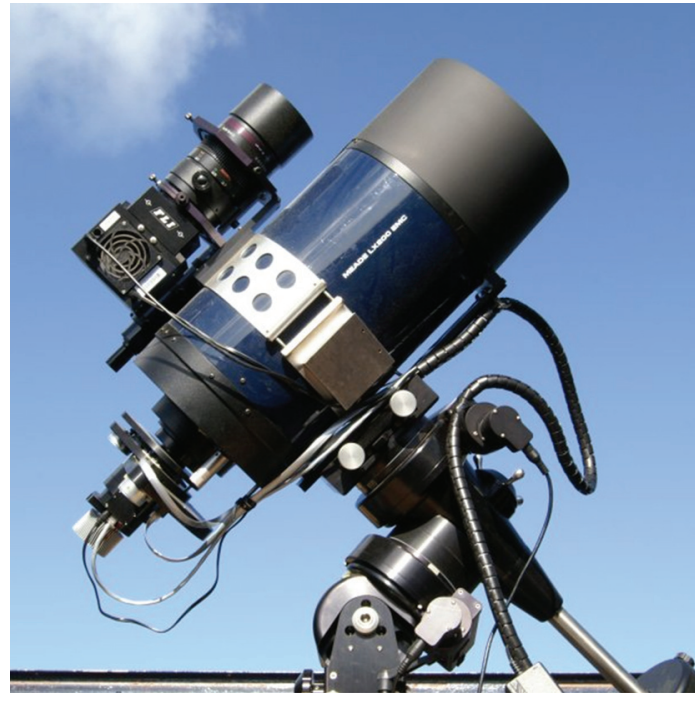

Figure 1: Burst Alert Robotic Telescope; wide field camera at left top.

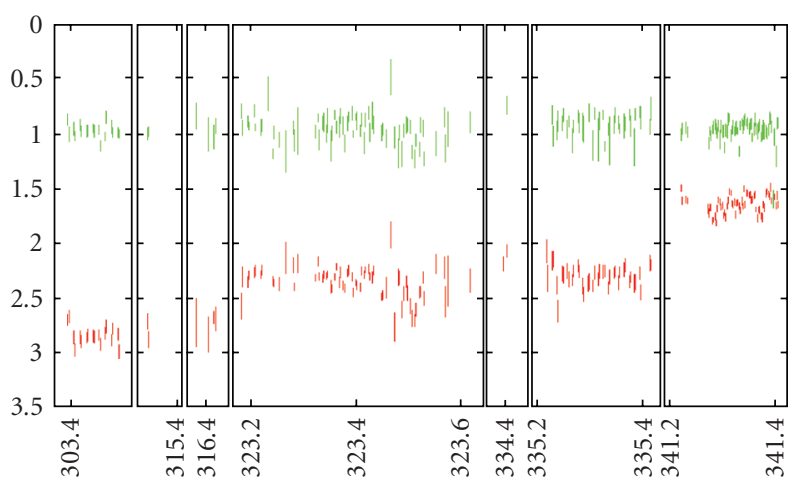

FIgUre 2: An example of photometric light curve of GK Per obtained from BART's data; $x$-axis shows time in JD-2453000.0.

shorter than one hour after an alert in years 2005-2007. BART has been observing more high energy sources, for example cataclysmic variable star GK Per. Its light curve is shown in the Figure 2. There were published numerous papers about BART, mostly dealing with GRBs, telescope setup, RTS2 and observational results [6-15] and a few with respect to another type of high energy objects $[16,17]$. 
TABLE 2: Overview of BART GRB reactions 2002-2005.

\begin{tabular}{lccl}
\hline GRB & GRB $\Delta$ & GCN $\Delta$ & comment \\
\hline 020124 & $11 \mathrm{~h} 30 \mathrm{~m}$ & $10 \mathrm{~h} 20 \mathrm{~m}$ & $\mathrm{R}>14 \mathrm{mag}$ \\
020305 & $10 \mathrm{~h} 30 \mathrm{~m}$ & $30 \mathrm{~m}$ & $\mathrm{R}>14 \mathrm{mag}$ \\
020317 & $58 \mathrm{~m}$ & $90 \mathrm{~s}$ & $\mathrm{R}>13,5 \mathrm{mag}$ \\
020331 & $5 \mathrm{~h} 30 \mathrm{~m}$ & $5 \mathrm{~h} 20 \mathrm{~m}$ & $\mathrm{R}>12,5 \mathrm{mag}$, bad weather \\
030824 & $1 \mathrm{~h} 50 \mathrm{~m}$ & $30 \mathrm{~m}$ & $\mathrm{R}>14,3 \mathrm{mag}$ \\
$031111 \mathrm{~A}$ & $1 \mathrm{~h} 10 \mathrm{~m}$ & $30 \mathrm{~s}$ & disturbing Moon \\
$031111 \mathrm{~B}$ & $10 \mathrm{~m}$ & $30 \mathrm{~s}$ & $\mathrm{R}>14,5 \mathrm{mag}, \mathrm{I}>14,2 \mathrm{mag}$ \\
040403 & $93 \mathrm{~m}$ & - & preburst limit \\
040606 & $5 \mathrm{~h} 30 \mathrm{~m}$ & - & $\mathrm{I}>12 \mathrm{mag}$ \\
050505 & $9,5 \mathrm{~m}$ & $29 \mathrm{~s}$ & $\mathrm{~V}>11,5 \mathrm{mag}$, poor cond. \\
$050509 \mathrm{~A}$ & $79 \mathrm{~s}$ & $62 \mathrm{~s}$ & $\mathrm{~V}>14,9 \mathrm{mag}$ \\
050520 & $15 \mathrm{~m}$ & $14 \mathrm{~m}$ & $\mathrm{~V}>14 \mathrm{mag}$ \\
050525 & $10 \mathrm{~m}$ & $16 \mathrm{~s}$ & $\mathrm{~V} \sim 15 \mathrm{mag}$ \\
050824 & $10 \mathrm{~m}$ & $31 \mathrm{~s}$ & $\mathrm{~V}>12 \mathrm{mag}$ \\
\hline
\end{tabular}

TABLE 3: Overview of BART GRB reactions 2005-2007.

\begin{tabular}{|c|c|c|}
\hline GRB & GRB $\Delta$ & GCN $\Delta$ \\
\hline GRB 070613 & $53.927 \mathrm{~s}$ & $21.539 \mathrm{~s}$ \\
\hline GRB 070502 & $01 \mathrm{~m} 42.696 \mathrm{~s}$ & $27.092 \mathrm{~s}$ \\
\hline GRB 070429 & $13 \mathrm{~m} \mathrm{35.885s}$ & $12 \mathrm{~m} 05.614 \mathrm{~s}$ \\
\hline GRB 070412 & $11 \mathrm{~m} 21.490 \mathrm{~s}$ & $07.903 \mathrm{~s}$ \\
\hline GRB 070411 & $52.225 \mathrm{~s}$ & $12.446 \mathrm{~s}$ \\
\hline GRB 070411 & $01: 03: 42.425$ & $58 \mathrm{~m} 26.233 \mathrm{~s}$ \\
\hline GRB 070406 & $19: 50: 48.262$ & $04.412 \mathrm{~s}$ \\
\hline GRB 070314 & $56.758 \mathrm{~s}$ & $37.045 \mathrm{~s}$ \\
\hline GRB 070306 & $54 \mathrm{~m} 11.582 \mathrm{~s}$ & $53 \mathrm{~m} 07.358 \mathrm{~s}$ \\
\hline GRB 061019 & $01 \mathrm{~m} 10.642 \mathrm{~s}$ & $33.251 \mathrm{~s}$ \\
\hline GRB 060929 & $02 \mathrm{~m} 36.382 \mathrm{~s}$ & $02 \mathrm{~m} 33.236 \mathrm{~s}$ \\
\hline GRB 060926 & $57.920 \mathrm{~s}$ & $44.080 \mathrm{~s}$ \\
\hline GRB 060901 & $04: 02: 02.340$ & $04 \mathrm{~m} 54.359 \mathrm{~s}$ \\
\hline GRB 060522 & $02 \mathrm{~m} 36.118 \mathrm{~s}$ & $32.778 \mathrm{~s}$ \\
\hline GRB 060512 & $13 \mathrm{~m} 44.794 \mathrm{~s}$ & $33.761 \mathrm{~s}$ \\
\hline GRB 060507 & $01 \mathrm{~m} 00.563 \mathrm{~s}$ & $40.254 \mathrm{~s}$ \\
\hline GRB 060421 & $33.695 \mathrm{~s}$ & $19.397 \mathrm{~s}$ \\
\hline GRB 051112 & $06 \mathrm{~m} \mathrm{49.959s}$ & $02 \mathrm{~m} 11.065 \mathrm{~s}$ \\
\hline GRB 051109 & $49.531 \mathrm{~s}$ & $21.104 \mathrm{~s}$ \\
\hline GRB 051013 & $-06 \mathrm{~m} 21.183 \mathrm{~s}$ & $16 \mathrm{~m} 36.237 \mathrm{~s}$ \\
\hline GRB 051011 & $01 \mathrm{~m} 05.765 \mathrm{~s}$ & $22.718 \mathrm{~s}$ \\
\hline GRB 051011 & $17 \mathrm{~m} 50.197 \mathrm{~s}$ & $31.649 \mathrm{~s}$ \\
\hline GRB 051011 & $01 \mathrm{~m} 06.120 \mathrm{~s}$ & $44.372 \mathrm{~s}$ \\
\hline GRB 051008 & $11 \mathrm{~m} 58.140 \mathrm{~s}$ & $21.769 \mathrm{~s}$ \\
\hline GRB 050922 & $05 \mathrm{~m} 57.392 \mathrm{~s}$ & $02 \mathrm{~m} 24.348 \mathrm{~s}$ \\
\hline GRB 050807 & $27.617 \mathrm{~s}$ & $20.170 \mathrm{~s}$ \\
\hline
\end{tabular}

\section{Conclusions}

BART is a good instrument for monitoring of objects with limiting magnitude 17.5 in R filter and for student's practice. RTS2 is well designed to take advantage of networking. There is certain probability to observe an optical counterpart of GRB considering a weather in our region and limiting magnitude of BART.

\section{Acknowledgments}

The authors acknowledge support by ESA PECS INTEGRAL Project 98023 and by the Grant Agency of the Czech Republic 205/08/1207. The third author was supported by Spanish Ministry of Education and Science Grant no. AP2003-1407. The authors would like thank everyone who help with BART for his/her help.

\section{References}

[1] P. Kubánek, M. Jelínek, S. Vítek, A. De Ugarte Postigo, M. Nekola, and J. French, "RTS2: a powerful robotic observatory manager," in Advanced Software and Control for Astronomy, $\mathrm{H}$. Lewis and A. Bridger, Eds., vol. 6274 of Proceedings of SPIE, Orlando, Fla, USA, May 2006, 62741V.

[2] P. Kubánek, "Remote Telescope System-2nd version," November 2008, http://rts2.org.

[3] P. Kubánek, M. Jelínek, M. Nekola, et al., "RTS2-remote telescope system, 2nd version," in Gamma-Ray Bursts: 30 Years of Discovery: Gamma-Ray Burst Symposium, vol. 727 of AIP Conference Proceedings, pp. 753-756, 2004.

[4] GCN, "The Gamma-Ray bursts Coordinates Network," November 2009, http://gcn.gsfc.nasa.gov.

[5] GCN circulars 1236, 1265, 1278, 2456, 2491, 3431, 3481, $3498,3500,3854,4319,4535,4536,4617,4675,4986$, 5092, 5658, 6335, 7651, 7689, 8092, November 2009, http://gcn.gsfc.nasa.gov/gcn3_archive.html.

[6] P. Kubánek, M. Jelínek, S. Vítek, et al., "Status of robotics telescopes driven by RTS2 (BART, BOOTES, FRAM and Watcher)," Nuovo Cimento della Societa Italiana di Fisica B, vol. 121, no. 12, pp. 1501-1502, 2006.

[7] P. Kubánek, M. Jelínek, R. Hudec, M. Nekola, and J. Štrobl, "BART: real time follow-up of GRBs since 2001," in GammaRay Bursts in the Swift Era: Sixteenth Maryland Astrophysics Conference, vol. 836 of AIP Conference Proceedings, pp. 684687, 2006.

[8] M. Jelínek, P. Kubánek, R. Hudec, M. Nekola, M. Topinka, and J. Strobl, "BART_-burst alert robotic telescope," in The Astrophysics of Cataclysmic Variables and Related Objects, J.-M. Hameury and J.-P. Lasota, Eds., vol. 330 of Proceedings of ASP Conference, p. 481, Astronomical Society of the Pacific, 2005.

[9] M. Jelínek, P. Kubánek, and M. Nekola, "BART 2001-2004: an intelligent robotic observatory," Nuovo Cimento della Societa Italiana di Fisica C, vol. 28, no. 4-5, pp. 735-738, 2005.

[10] M. Jelínek, P. Kubánek, M. Nekola, and R. Hudec, "BART: an intelligent GRB and sky monitoring telescope (2000-2004)," Astronomische Nachrichten, vol. 325, no. 6-8, p. 678, 2004.

[11] M. Jelínek, P. Kubánek, M. Nekola, and R. Hudec, "GRB optical prompt followup with BART," in Third Rome Workshop on Gamma-Ray Bursts in the Afterglow Era, M. Feroci, F. Frontera, N. Masetti, and L. Piro, Eds., vol. 312 of ASP Conference Series, p. 213, Astronomical Society of the Pacific, 2004.

[12] M. Jelínek, R. Hudec, P. Kubánek, et al., "BART-recent status," in Gamma-Ray Burst and Afterglow Astronomy 2001: A Workshop Celebrating the First Year of the HETE Mission, vol. 662 of AIP Conference Proceedings, pp. 520-522, 2003.

[13] M. Jelínek, P. Kubánek, M. Nekola, et al., Robotic Telescope $B A R T$, vol. 16, Odessa Astronomical, 2003. 
[14] R. Hudec, M. Nekola, P. Kubánek, C. Polášek, and A. J. CastroTirado, "The status of the ondrejov BART experiment," in Gamma-Ray Bursts in the Afterglow Era, E. Costa, F. Frontera, and J. Hjorth, Eds., Eso Astrophysics Symposia, p. 396, Springer, London, UK, 2001.

[15] R. Hudec, J. Soldán, V. Hudcová, et al., "Optical observations of GRBs: EN, BART, and OMC," in Proceedings of the 5th Huntsville Symposium on Gammaray Bursts, vol. 526 of AIP Conference Proceedings, pp. 265-269, September 2000.

[16] J. Soldán, R. Hudec, M. Němeček, and T. Rezek, "Burst alert robotic telescope (BART) and monitoring of selected AGNs," in BL Lac Phenomenon Poster Session, p. 103, Turku, Finland, June 1998.

[17] R. Hudec, M. Jelínek, M. Nekola, and P. Kubánek, "BART robotic telescope and blazar monitoring," in High Energy Blazar Astronomy, L. Takal and E. Valtaoja, Eds., vol. 299 of ASP Conference Series, pp. 193-196, Astronomical Society of the Pacific, San Francisco, Calif, USA, 2003. 

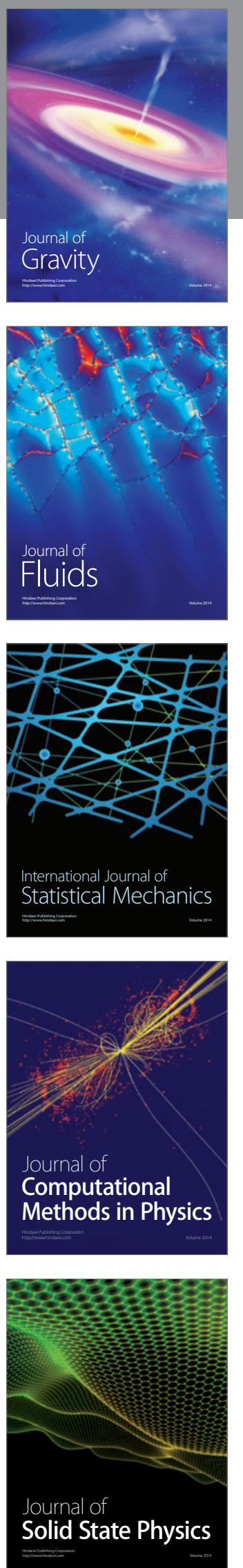

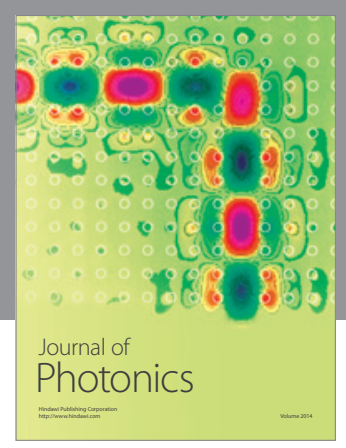

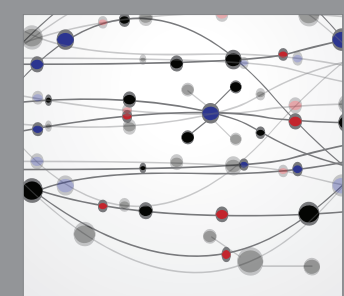

The Scientific World Journal
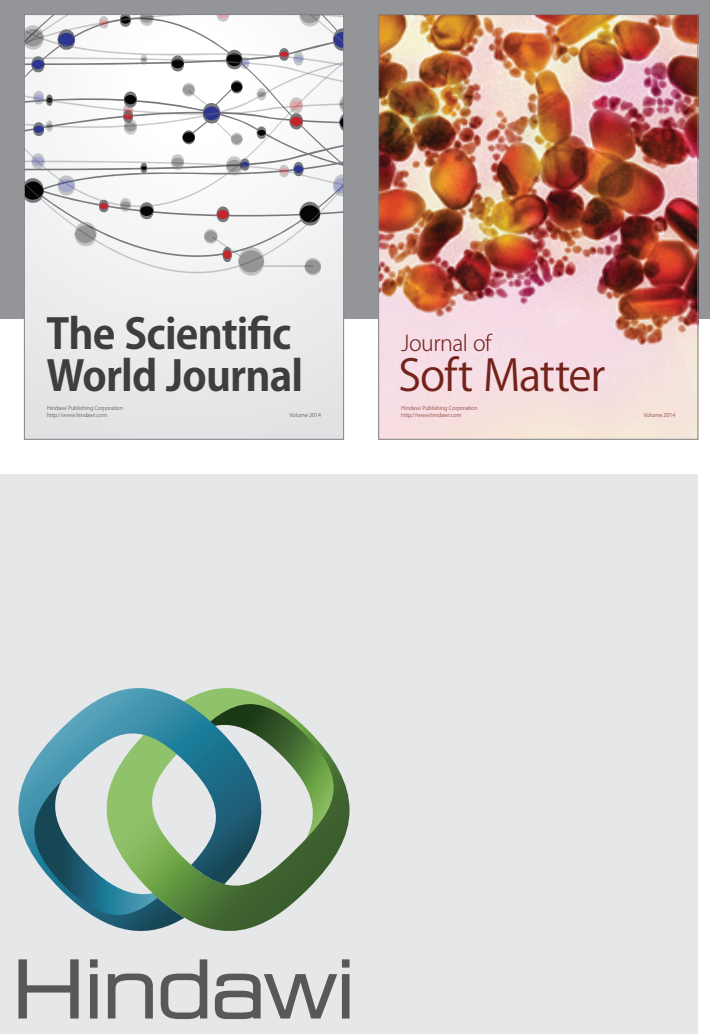

Submit your manuscripts at

http://www.hindawi.com
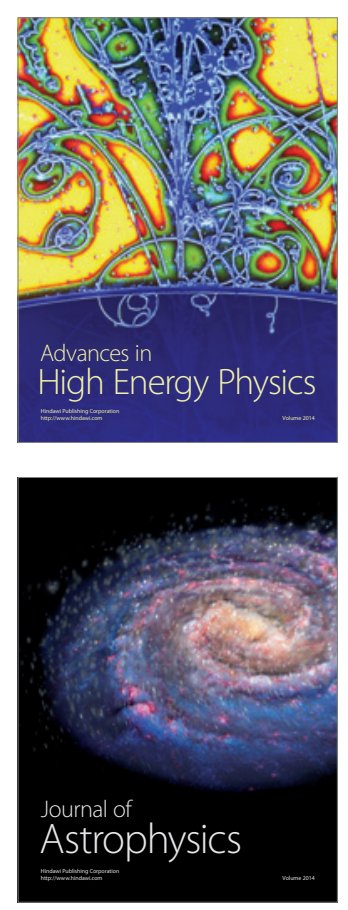
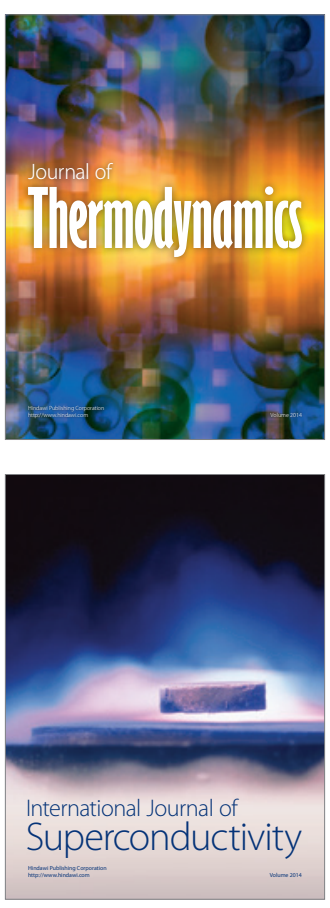
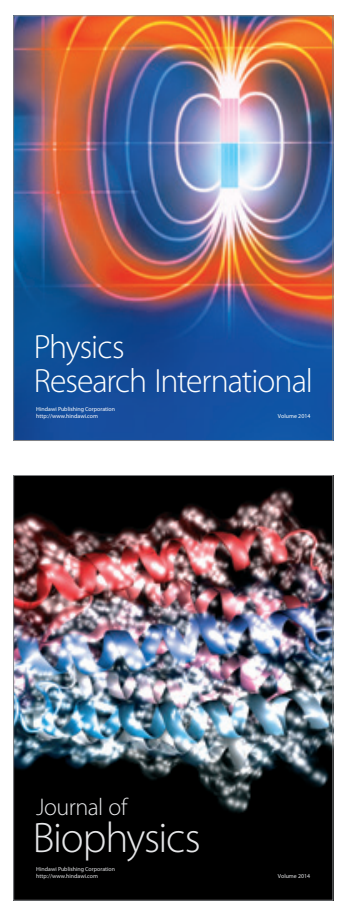
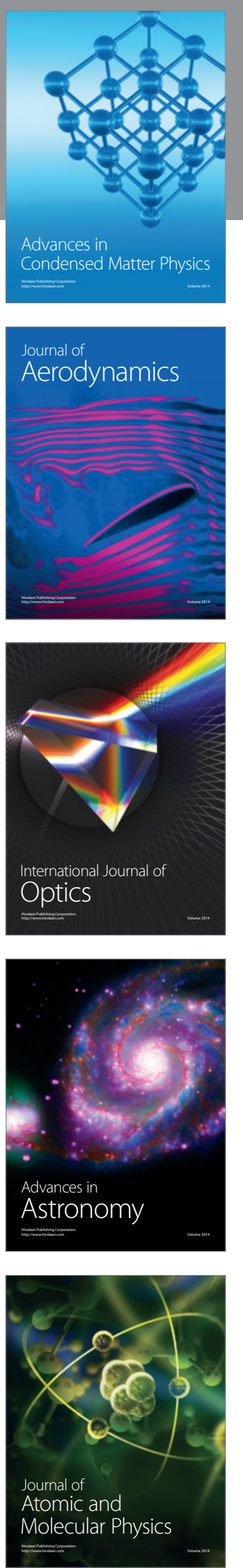